\title{
BMJ Open Atorvastatin for prevention of disease progression and hospitalisation in liver cirrhosis: protocol for a randomised, double-blind, placebo-controlled trial
}

\author{
Nina Kimer (1D , , ${ }^{1,2}$ Henning Grønbæk, ${ }^{3}$ Rikard Gøran Fred, ${ }^{2}$ Torben Hansen, ${ }^{2}$ \\ Atul Shahaji Deshmukh, ${ }^{4}$ Mathias Mann, ${ }^{4,5}$ Flemming Bendtsen ${ }^{1}$
}

To cite: Kimer N, Grønbæk H, Fred RG, et al. Atorvastatin for prevention of disease progression and hospitalisation in liver cirrhosis: protocol for a randomised, double-blind, placebocontrolled trial. BMJ Open 2020;10:e035284. doi:10.1136/ bmjopen-2019-035284

- Prepublication history for this paper is available online. To view these files, please visit the journal online (http://dx.doi. org/10.1136/bmjopen-2019035284).

Received 25 October 2019 Revised 09 December 2019 Accepted 08 January 2020

\section{ABSTRACT}

Introduction Patients with liver cirrhosis are often diagnosed late and once complications are present, the 2-year survival is $50 \%$. Increasing evidence supports systemic inflammation and metabolic dysfunction in the hepatic stellate cell as key drivers of progression of cirrhosis. However, there is no registered medication, that targets inflammation and cellular dysfunction in the liver.

Methods and analysis In a randomised double-blind and placebo-controlled trial with atorvastatin for liver cirrhosis, we aim to investigate clinical endpoints of survival, hospitalisations and safety, but also exploratory endpoints of genomics and protein functions in the liver.

Ethics and dissemination There is no registered medication that actively prevents development of complications or systemic inflammation in liver cirrhosis All patients continue regular clinical management during the trial period. Atorvastatin has been on the market for several years with a safety profile that is acceptable even in patients with liver disease. A beneficial effect of atorvastatin on clinical outcomes in cirrhosis will provide cheap and effective causal treatment for chronic liver disease. The trial is registered by the Danish Data Protection Agency (P-2019-635) and approved by the Danish Medicines Agency (EudraCT 2019-001806-40) and the Scientific Ethics Committee of the Capital Region of Denmark (H-19030643) before initiation. Reporting of the trial will follow the Consolidated Standards of Reporting Trials guidelines for reporting of randomised clinical trials. Trial registration number The trial is registered in clinicaltrials.gov (NCT04072601) and in clinicaltrialsre gister.eu (EudraCT 2019-001806-40) (Pre-results).

\section{INTRODUCTION}

In Denmark, the incidence of liver cirrhosis has increased fivefold for men and tripled for women over the last 40 years. Approximately 1600 people are diagnosed with liver cirrhosis every year. ${ }^{1}$ Patients readmitted to hospital have higher 90 -day mortality than those who avoid readmission. ${ }^{2}$

The high incidence of liver cirrhosis in Denmark is caused by a combination of genetic susceptibility, a wide use of alcohol

\section{Strengths and limitations of this study}

- The trial is double blind and placebo controlled

- The trial has primary clinical endpoints of mortality and hospitalisation.

- The trial has secondary endpoints in exploratory studies of liver dysfunction and disease progression.

- The trial applies novel omics techniques in decompensated liver disease.

- The trial design includes 18 months of treatment and up to 5 years of follow-up.

and a population increasing in age and body mass index. Patients with liver cirrhosis are often diagnosed late and once complications are present, the 2-year survival is $50 \% .^{3}$ Systemic inflammation predisposes to an excess risk of infections and mortality. ${ }^{4}$

Treatment of liver cirrhosis has focused on addressing isolated complications and not on causal factors driving the development and progression of the disease. The scientific literature is characterised by only few randomised trials evaluating new pharmacological treatment principles. $^{5-7}$ In the past decade, increasing evidence supports systemic inflammation and metabolic dysfunction in the hepatic stellate cell as key drivers of progression of cirrhosis. ${ }^{89}$ Only a few studies assess combination therapy with potential effects on inflammation in the liver, on portal venous blood pressure and kidney function, and evidence is scarce in this area. ${ }^{10}$ At present, there is no registered medication that targets inflammation and cellular dysfunction in the liver.

Use of statins (3-hydroxy-3-methylglutarylcoenzyme A reductase inhibitors) in cirrhosis may reduce the risk of complications and mortality. ${ }^{11} 12$

Several studies have demonstrated the beneficial effects of statins in vascular and 
heart disease. ${ }^{13} 14$ Statins have antithrombotic effects, decrease oxidative stress and inflammation at the vessel wall, and improve endothelial dysfunction by increasing nitric oxide (NO) production in endothelial cells. ${ }^{15-17}$ In cirrhosis, endothelial NO release is impaired in the liver microvasculature and is a major contributor to the increased hepatic resistance and development of portal hypertension. ${ }^{18} 19$

In rodent models, statins are shown to decrease intrahepatic vascular resistance and improve flow-mediated vasodilation of liver vasculature in the cirrhotic liver. These effects are mediated by an upregulation of NO production at the liver vasculature through an enhancement in endothelial NO synthase activity. ${ }^{2021}$ Statins seems also to inhibit fibrogenesis in cirrhotic rats. ${ }^{22}$ In recent years, a series of pilot studies have assessed the effects of simvastatin on portal hypertension. ${ }^{523-25}$

Lately, a large randomised trial showed an effect of simvastatin on mortality, but not on rebleeding in patients with cirrhosis and previous variceal bleeding. ${ }^{12}$ In a Danish national registry study, use of statins reduced the risk of mortality among patients with cirrhosis. ${ }^{26}$ Evidence supporting the use of statins in a real-world clinical setting, and data on the effects on inflammation and generation of fibrosis in the liver in humans is in high demand.

Atorvastatin is a potent and widely used drug with less toxicity and a better safety profile than other statins. ${ }^{13} 27$ It has demonstrated inhibitory effects on development of cirrhosis and hepatocellular carcinoma as well as similar beneficial effects on portal hypertension. ${ }^{2829}$ Despite these promising effects, international experts agree that further randomised controlled trials in large series of patients with hard clinical endpoints should be performed before statins can be recommended for use in clinical practice. ${ }^{30}$

Mass spectrometry-based proteomics technology is constantly improving. In 2016, a robust and scalable sample preparation technology for the human plasma proteome was developed, and an inflammatory protein pattern was defined and quantified dynamically in a clinical trial. ${ }^{31}{ }^{32}$ Recent advances now allow rapid quantification of a complete proteome in simple organisms and near exhaustive proteomes of mammalian cells. ${ }^{33} 34$ Bioinformatics analysis is continuously evolving, ${ }^{35} 36$ and have in recent years moved in to the field of translational research. Performance of proteomics on human liver cells with advanced disease and with systemic inflammation will allow us to explore drug efficacy and pharmacodynamics at the highest level available, protein transcription and expression in the hepatic cells.

Genome-wide association studies have identified large numbers of single cell nucleotide polymorphisms associated to insulin resistance, and other metabolic traits related to metabolism and progression of liver fibrosis. ${ }^{37}{ }^{38}$ From whole genome sequence data, information on both rare variants and common single nucleotide polymorphisms (SNPs) can be extracted, and a global reference system for relevant traits can be defined and

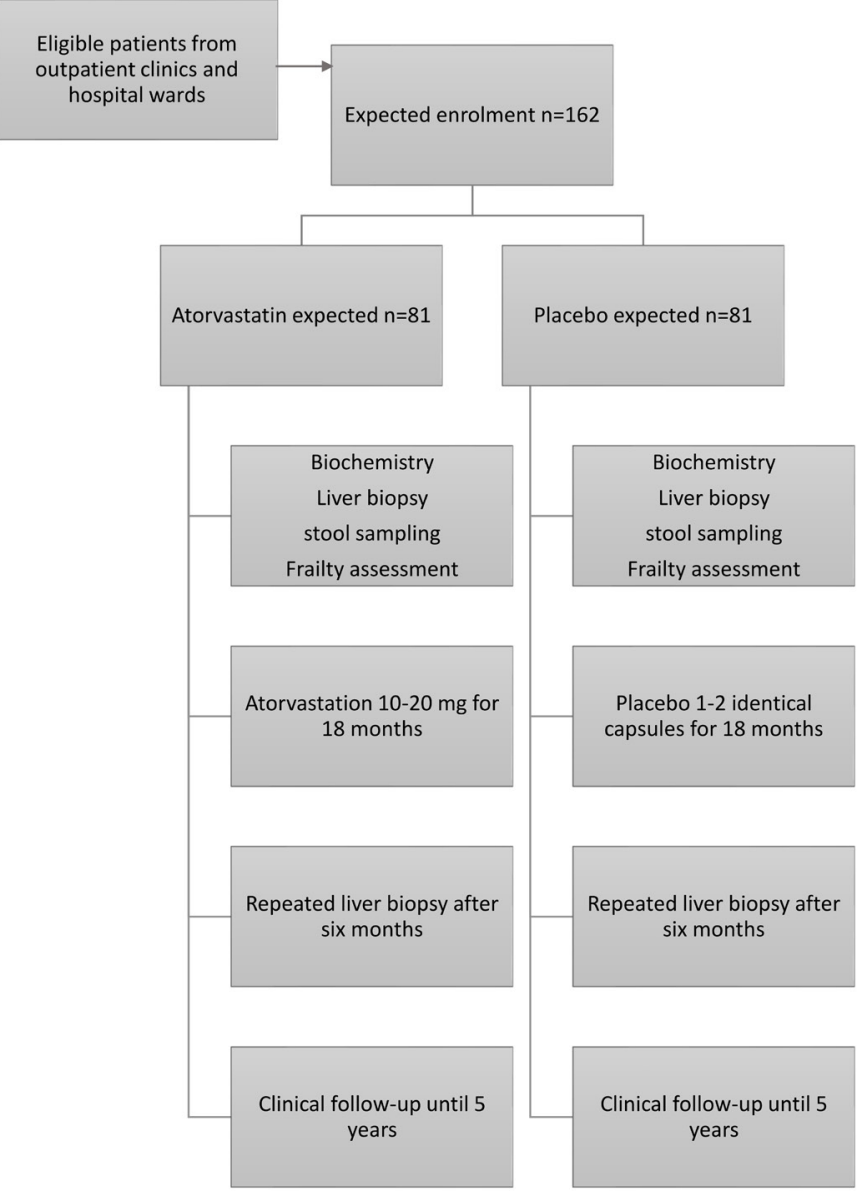

Figure 1 Trial flow.

used in development of risk algorithms. Recently developed single-cell RNA-sequencing methods and cell-free DNA sequencing enables unbiased and high-throughput transcriptomic analyses of individual cells and an estimate of turnover of individual cell types in the liver. ${ }^{39} 40$

This trial will investigate whether atorvastatin improves survival or delays onset of systemic inflammation and decompensation in cirrhosis. Secondarily, the trial will assess whether macrophage activation, immune response or protein expression in the hepatic stellate cell are altered by atorvastatin treatment.

\section{METHODS AND ANALYSIS}

In a two-centre, randomised, double-blind and placebocontrolled clinical trial, participants will be allocated to atorvastatin $10-20 \mathrm{mg}$ daily or placebo in a 1:1 ratio. Study flow and investigations are stated in figure 1 . Initiation of the trial is planned for October 2019.

\section{Selection of participants}

The trial will take place in two university hospitals with referral from other hospitals, hospital departments and general practice. Patients referred to the outpatient clinics or hospitalised in the Gastro Unit, Medical Division, Copenhagen University Hospital Hvidovre and Department of Hepatology and Gastroenterology, 
Aarhus University Hospital, are eligible for inclusion. On average, 225 people are every year diagnosed with liver cirrhosis in these two departments which will supply for adequate inclusion. Patients will be offered enrolment within 3 months of diagnosis.

Patients with liver cirrhosis, diagnosed by liver biopsy, ultrasound or CT scan of the liver, and clinical biochemistry compatible with cirrhosis are eligible for inclusion. Participants must have portal hypertension with a hepatic venous pressure gradient measured by liver vein catheterisation $\geq 10 \mathrm{~mm} \mathrm{Hg}$. In women, documented absence of pregnancy and, unless in menopause, commitment to use adequate contraception. Participants must be able to read and understand project information in Danish and give written, informed consent to participation.

Patients are excluded if they are treated with statins within the last year; are pregnant or breast feeding; have hepatocellular carcinoma; HIV infection and receive treatment with protease inhibitors; or Model for endstage liver disease (MELD) score above 23 or Child-Pugh score higher than 13 .

Patients with liver cirrhosis and a clinically verified infection (standard biochemistry, culture) within the last 4 weeks; patients with clinical and biochemical signs of hepatorenal syndrome defined by current guidelines within the last 14 days $^{41}$; patients with hepatic encephalopathy grade 2 or higher; and patients in whom the clinician and investigators may have reason to doubt compliance to trial medication will also be excluded.

Dyslipidaemia is not an exclusion criterion. However, treatment effects on cholesterol levels will not be monitored during the trial but will be measured by routine lab and blinded to investigators and clinical personnel.

\section{Interventions}

The intervention group will receive atorvastatin $10 \mathrm{mg}$ capsules daily for 78 weeks in addition to standard treatment. Dose will be adjusted to $20 \mathrm{mg}$ after 4 weeks of treatment if tolerated by the trial participant.

The control group will receive placebo capsules, identical in shape, size and colour to atorvastatin $10 \mathrm{mg}$, one capsule daily for 78 weeks in addition to standard treatment. Dose will be adjusted to two capsules daily after 4 weeks of treatment, if the trial participant tolerates it.

Discontinuation of trial drug will be performed in case of: the participant withdraws informed consent; the participant reach a primary endpoint (death or liver transplantation); the participant experiences serious adverse events that cause a risk to the participant's life or viability; the participant develops toxicity, intolerance or allergy associated with intervention medication; the trial participant is admitted to hospital for other morbidity that is incompatible with further treatment with atorvastatin; or blinding is revealed.

Physicians with training in Good Clinical Practice (GCP) will perform specific tasks of information, screening and obtaining informed consent. Staff physicians and project nurses will secure adequate recruitment. In case of slow recruitment, a third investigating site will be opened (Copenhagen University Hospital Rigshospitalet). Participants give informed consent to participation and separate informed consent to storage of biological material, as required by Danish Legislation.

\section{Assignment of interventions}

Randomisation is computer generated in block randomisation of every six patients. The randomisation list is generated by The Regional Pharmacy, University Hospital Herlev, who packages and blinds medication. Participants are allocated to treatment by a unique study drug ID (consecutive numbers). Each number refers to a study drug (active or placebo). The randomisation and identification lists are stored at The Regional Pharmacy, University Hospital Herlev. To achieve complete blindness of trial participants and personnel, a comparator similar in size, shape and colour is used.

Atorvastatin tablets $10 \mathrm{mg}$ can be repackaged into capsules with flavourless and colourless filler, and a placebo capsule with only filler that has no medicinal or biological effects is manufactured.

The sponsor, principal investigator, subinvestigators and all personnel handling patients in daily clinic will be blinded to interventions. One laboratory pharmacist responsible for packing and blinding of medication will be unblinded. This person is physically located at The Regional Pharmacy, University Hospital Herlev and is not involved in trial specific routines. A series of envelopes produced by the above mentioned unblinded pharmacist with each identification number will allow for unblinding of each individual participant if necessary.

Study drugs will be distributed to participants at baseline, at 1 and 3 months and thereafter every 3 months. Participants are asked to bring back remaining tablets and containers for registration every 3 months. Returned number of tablets are registered. Accounting of study medication is performed after 6 and 18 months for evaluation of adherence to medication.

\section{Sample size}

The following is based on data from a previous Danish cohort of patients with liver cirrhosis and decompensation at the time of diagnosis and recent studies of simvastatin in cirrhosis. ${ }^{312}$

Prior data indicate a median survival time between 13 and 48 months, depending on the progression of liver disease and type of decompensation. ${ }^{3}$ A former trial in a population of patients with decompensated cirrhosis reported a relative risk reduction to 0.39 in the statin group. $^{12}$

A conservative sample size estimate is therefore based on the following trial conditions: (1) one control per experimental subject, (2) an inclusion period of 24 months, (3) an additional follow-up after end of inclusion of 24 months.

Prior data may indicate that the median survival time on the control treatment is 35 months. If the true HR 


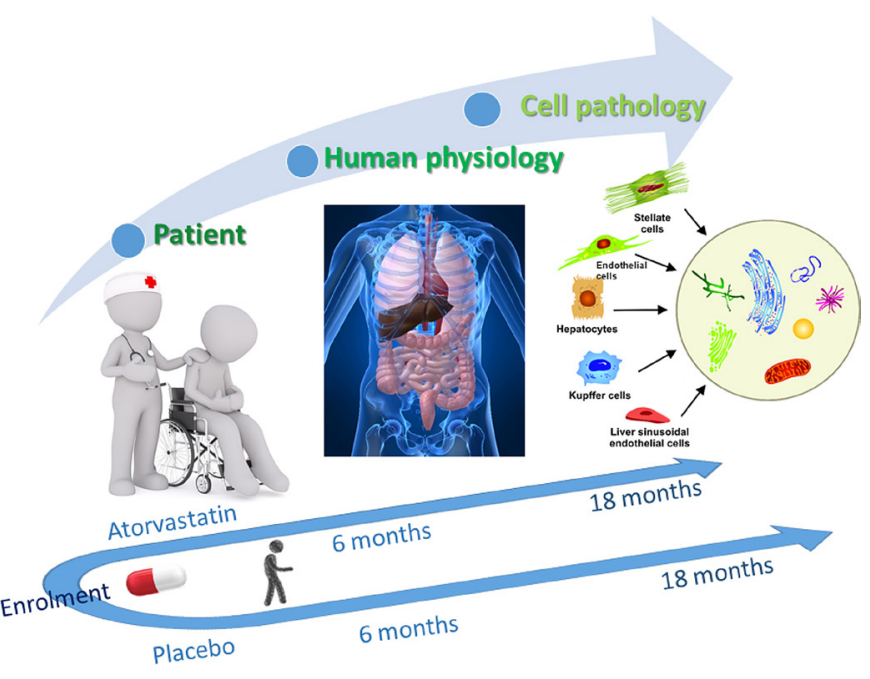

Figure 2 Graphical abstract.

of control subjects relative to experimental subjects is 0.52 , we will need to study 70 experimental subjects and 70 control subjects to reject the null hypothesis that the experimental and control survival curves are equal with a probability of 0.85 . The type I error probability associated with the test of this null hypothesis is 0.05 .

With an expected dropout rate of $15 \%$, we plan to enrol 162 participants.

\section{Outcomes}

The clinical trial will apply a three-dimensional approach in investigating the mechanisms of atorvastatin in cirrhosis: (1) the patient level, (2) the human physiology level and (3) the cellular level (see figure 2, graphical abstract).

The primary endpoints are: composite endpoint of numbers of death or liver transplantation after 1.5 years, and hospitalisation with liver related complications after
1.5 years. Secondary endpoints on the patient level are: (1) survival (2) adverse events, (2) decompensation of liver cirrhosis and time to decompensation, (3) clinical scores of Child and MELD, (4) frailty evaluated by the Life Space Assessment Questionnaire and the Short Physical Performance Battery. ${ }^{42}$

Further, on the human physiology level, exploratory endpoints are: impact on inflammation and macrophage activation measured by immunohistochemistry of the stellate cell in combination with PCR analysis for specific markers of inflammation in blood, macrophage activation and haemodynamic investigations of portal hypertension and effect on hepatic venous pressure gradient.

Exploratory endpoints on the cellular level: (1) Cellular activation. Gene activation by transcriptomics of mRNA on the hepatic stellate cell. Gene analyses and single cell transcriptomics allow for investigation of gene activity and inclusion of genetic traits in risk prediction models for disease progression. (2) Protein activity in the hepatic stellate cell. By high-sensitivity Mass spectrometry-based proteomics, we will perform analysis of hepatic stellate cells and Kupffer cells under atorvastatin influence. (3) Characterisation of the human microbiome with shotgun metagenomic sequencing of all DNA, at a depth of 30 million paired end reads ( $9 \mathrm{~GB}, 2 \times 150 \mathrm{bp}$ ), and association to disease progression will be conducted. Since microbiome analysis and omics technologies are rapidly evolving, a detailed plan for analysis will be conducted after baseline sampling, to ensure low cost and resource efficiency.

The investigational programme is stated in table 1 . For the exploratory outcomes of human physiology and cell pathology, a biobank containing blood, liver tissue and stool is created.

Table 1 Investigational programme

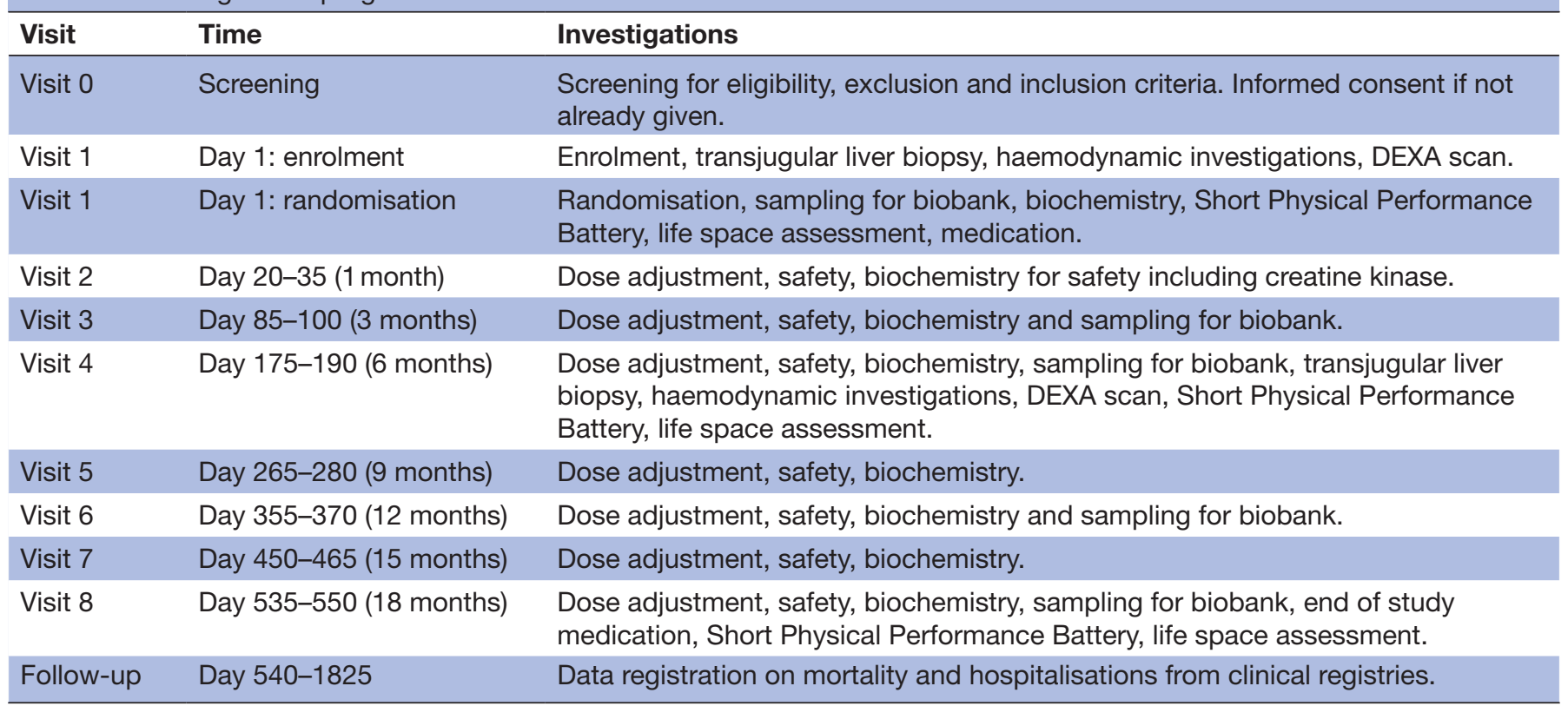




\section{Safety}

Initial dose of study drug is $10 \mathrm{mg}$ which can be increased to $20 \mathrm{mg}$ after 2-4 weeks if the participant does not develop side effects. Creatine kinase and standard biochemistry including liver enzymes are measured for safety reasons after 2 weeks, to rule out myositis and liver damage. Side effects and events are consecutively registered throughout the trial.

\section{Data analysis and data management}

The primary outcomes will be analysed as a time to event variable, and groups are compared with the stratified logrank test and HR by using the Cox model adjusting by the clinical scores MELD and Child. Survival will be analysed by the Kaplan-Meier method.

For continuous outcome variables, a mixed model analysis or Generalized Estimating Equations will be applied. Adjustment for baseline differences will be taken into account by a longitudinal analysis of covariance. Continuous variables measured repeatedly over time is analysed through mixed models for repeated measurements.

Omics technologies are developing rapidly and statistical methods for handling big data evenly. A complete data analysis plan will be performed before initiation of the exploratory analyses.

Statistical analyses will be performed as per protocol and as intention to treat analyses. Missing data will not be replaced but left blank in statistical analysis. For intention to treat analyses on repeated measures, the last available value will be carried forward to last value.

Data are stored in a Redcap database. The trial is monitored by the GCP Unit, University of Copenhagen, and site-specific data managers. The trial follows the guidelines of International Conference of Harmonization - Good Clinical Practice (ICH-GCP), and the Trial Master File will serve as source for Standard Operating Procedures, data agreements, contracts, study guide and communications between sites. Definition and handling of adverse events follow the ICH-GCP guidelines.

\section{ETHICS AND DISSEMINATION}

\section{Patient and public involvement}

Patients or the public were not involved in trial design. The authors adhere to the Ethical guidelines of the Declaration of Helsinki, aiming to protect the life, health, dignity, integrity, right to self-determination, privacy and confidentiality of personal information of research subjects.

The present clinical trial will assess possible effects of adding atorvastatin to standard treatment in preventing disease progression and complications to liver cirrhosis. Hence, trial participants who choose to participate in the trial will receive standard of care according to relevant medical guidelines for treatment and prevention of complications to liver cirrhosis. ${ }^{41}$

Half of participants will receive placebo medicine. At present, there is no registered medication that prevents systemic inflammation or actively prevents development or onset of complications to liver cirrhosis, and it is yet to be proven if statins have effects on these parameters. Participants are not withheld active medication. All patients continue their regular prescribed medication during the trial period.

Former clinical and basic science suggest that statin treatment may ameliorate the systemic and local inflammation cascade involved in the progression of liver cirrhosis and complications hereto. ${ }^{213044}$ Addition of atorvastatin to standard treatment may improve survival and may decrease the risk of developing complications to liver cirrhosis, such as delaying onset of ascites, variceal bleeding or delaying development of sarcopenia and hepatic encephalopathy in any degree. Also, the clinical trial will provide a better understanding of disease progression on a cellular level in stellate cells and Kupffer cells in the liver, as well as insight into which mechanisms drives the inflammatory response involved in progression from fibrosis to cirrhosis in the liver. However, actual clinical and patient-relevant benefits of atorvastatin in cirrhosis are still uncertain.

Participants in the trial may experience extra discomfort in relation to having a second liver biopsy done after 6 months of treatment. These nuisances may be pain in relation to procedures and a minimal $(<0.9 \%)$ but extra risk of bleeding after procedures. ${ }^{45}$ Participants in the trial will be subject to monthly hospital visits for 3 months and then 3 monthly visits until end of trial. However, the regular follow-up as recommended for liver patients varies between every 14 days to yearly visits to hospital, and the disadvantage of participating in the clinical trial with regard to hospital visits is therefore difficult to assess. Combination of study visits and regular outpatient visits are pursued to minimise the time spent in hospital for participants.

The tolerability of statins has been evaluated in numerous trials in different patient populations. ${ }^{12} 1446$ Possible side effects are rare or very rare, and include abdominal pain, mild hepatitis, pancreatitis, anaemia, myopathies or peripheral neuropathies or allergic reactions. Meta-analyses have found that statins are equally safe as control treatment regarding adverse and serious adverse events, and that rates of discontinuation and the most frequent side effect myopathy were equal between simvastatin and placebo in clinical trials. ${ }^{14} 46$ Safety of statins in chronic liver disease has been assessed in only a few clinical trials, but reviews of observational studies report high safety and low risk of adverse events. ${ }^{10} 4748$

Recruitment of participants will follow Danish Legislation and data concerning trial participants will be protected under the Data Protection Regulation and the Act on Health (Sundhedsloven). Advertisement or recruitment will not be performed outside public hospitals.

\section{Dissemination of results}

Results on all outcomes will be sought published in international journals with peer review. A preliminary plan for dissemination prioritises data in to the following groups: primary endpoints including safety, inflammation and links to macrophage activation and gut 
microbiome; exploratory manuscripts on metagenomics and proteomics.

Listing of authors will follow the International Committee of Medical Journal Editors' recommendations for authorship. ${ }^{49}$

\section{Perspectives}

Atorvastatin has been on the market for several years with a safety profile that is acceptable even in patients with liver disease. Retrospective and register studies have evaluated the safety and risks of statins in cirrhosis. ${ }^{103050} \mathrm{~A}$ beneficial effect of atorvastatin on clinical outcomes in preventing disease progression and decompensation of cirrhosis will provide cheap and effective causal treatment directed at cirrhosis and fibrogenesis and not just symptom relief in decompensated liver disease.

\section{Author affiliations}

${ }^{1}$ Gastro Unit, Medical Division, Hvidovre Hospital, Hvidovre, Denmark

${ }^{2}$ Novo Nordisk Foundation Centre for Basic Metabolic Research, University of Copenhagen Faculty of Health and Medical Sciences, Kobenhavn, Denmark

${ }^{3}$ Department of Hepatology and Gastroenterology, Aarhus University Hospital, Aarhus, Denmark

${ }^{4}$ Clinical Proteomics Group, Proteomics Program, Novo Nordisk Foundation Center for Protein Research, University of Copenhagen, 2200 Copenhagen, Denmark ${ }^{5}$ Max-Planck-Institute of Biochemistry, Martinsried, Munich, Bayern, Germany

\section{Twitter Nina Kimer @NKimer}

Contributors NK and FB designed the trial. NK drafted the protocol. FB and HG revised the protocol for academic content. ASD, RGF, TH and MM revised the methodology sections on the exploratory outcomes. NK drafted the first version of the protocol manuscript. AD and RF revised the section on methods of exploratory outcomes. All authors critically revised the manuscript and approved of the final version of the manuscript.

Funding This work and the following trial were supported by the Danish Regions (Regionernes Medicin pulje) 2018 grant number EMN-2018-01114; and University Hospital Hvidovre Strategic Research Fund 2018. The clinical trial is further supported by a postdoctoral fellowship in Translational Medicine for Nina Kimer, via the Bridge Translational Excellence Program, University of Copenhagen 2019, (NNF 18SA0034956). Trial sponsor and initiator: Flemming Bendtsen, MD DmSC; Gastro Unit, Medical Division; University Hospital Amager-Hvidovre; Kettegaard Alle 30, 2650 Hvidovre, Denmark.

Competing interests None declared.

Patient consent for publication Not required.

Ethics approval The trial is approved by the Danish Medicines Agency (EudraCT 2019-001806-40), the Scientific Ethics Committee of the Capital Region of Denmark (H-19030643) and by the Danish Data Protection Agency (P-2019-635). In addition, the biobank for future research is registered by the Danish Data Protection Agency separately (P-2019-274).

Provenance and peer review Not commissioned; externally peer reviewed.

Open access This is an open access article distributed in accordance with the Creative Commons Attribution Non Commercial (CC BY-NC 4.0) license, which permits others to distribute, remix, adapt, build upon this work non-commercially, and license their derivative works on different terms, provided the original work is properly cited, appropriate credit is given, any changes made indicated, and the use is non-commercial. See: http://creativecommons.org/licenses/by-nc/4.0/.

ORCID iD

Nina Kimer http://orcid.org/0000-0002-4807-1575

\section{REFERENCES}

1 Jepsen P, Vilstrup H, Sørensen HT. Alcoholic cirrhosis in Denmark - population-based incidence, prevalence, and hospitalization rates between 1988 and 2005: a descriptive cohort study. BMC Gastroenterol 2008;8:3.

2 Berman K, Tandra S, Forssell K, et al. Incidence and predictors of 30-day readmission among patients hospitalized for advanced liver disease. Clin Gastroenterol Hepatol 2011;9:254-9.

3 Jepsen P, Ott P, Andersen PK, et al. Clinical course of alcoholic liver cirrhosis: a Danish population-based cohort study. Hepatology 2010;51:1675-82.

4 Clària J, Stauber RE, Coenraad MJ, et al. Systemic inflammation in decompensated cirrhosis: characterization and role in acute-onchronic liver failure. Hepatology 2016;64:1249-64.

5 Abraldes JG, Albillos A, Bañares R, et al. Simvastatin lowers portal pressure in patients with cirrhosis and portal hypertension: a randomized controlled trial. Gastroenterology 2009;136:1651-8.

6 Dhar A, Tschotazis E, Brown R, et al. LP11: Warfarin anticoagulation for liver fibrosis in patients transplanted for hepatitis C (WAFT-C): results at one year. J Hepatol 2015;62:S268-9.

7 Kimer N, Pedersen JS, Busk TM, et al. Rifaximin has no effect on hemodynamics in decompensated cirrhosis: a randomized, doubleblind, placebo-controlled trial. Hepatology 2017;65:592-603.

8 Krenkel O, Hundertmark J, Ritz TP, et al. Single cell RNA sequencing identifies subsets of hepatic stellate cells and myofibroblasts in liver fibrosis. Cells 2019;8:503.

9 Tsuchida T, Friedman SL. Mechanisms of hepatic stellate cell activation. Nat Rev Gastroenterol Hepatol 2017;14:397-411.

10 Kamal S, Khan MA, Seth A, et al. Beneficial effects of statins on the rates of hepatic fibrosis, hepatic decompensation, and mortality in chronic liver disease: a systematic review and meta-analysis. Am J Gastroenterol 2017;112:1495-505.

11 Bang UC, Benfield T, Bendtsen F. Reduced risk of decompensation and death associated with use of statins in patients with alcoholic cirrhosis. A nationwide case-cohort study. Aliment Pharmacol Ther 2017:APT0908-2017.R1.

12 Abraldes JG, Villanueva C, Aracil C, et al. Addition of simvastatin to standard therapy for the prevention of variceal rebleeding does not reduce rebleeding but increases survival in patients with cirrhosis. Gastroenterology 2016;150:e1163:1160-70.

13 Yebyo HG, Aschmann HE, Kaufmann M, et al. Comparative effectiveness and safety of statins as a class and of specific statins for primary prevention of cardiovascular disease: a systematic review, meta-analysis, and network meta-analysis of randomized trials with 94,283 participants. Am Heart J 2019;210:18-28.

14 Zhang W, Zhang Y, Li C-W, et al. Effect of statins on COPD: a metaanalysis of randomized controlled trials. Chest 2017;152:1159-68.

15 Kalinowski L, Dobrucki LW, Brovkovych V, et al. Increased nitric oxide bioavailability in endothelial cells contributes to the pleiotropic effect of cerivastatin. Circulation 2002;105:933-8.

16 Lefer DJ. Statins as potent antiinflammatory drugs. Circulation 2002;106:2041-2.

17 McGirt MJ, Lynch JR, Parra A, et al. Simvastatin increases endothelial nitric oxide synthase and ameliorates cerebral vasospasm resulting from subarachnoid hemorrhage. Stroke 2002;33:2950-6.

18 Gupta TK, Toruner M, Chung MK, et al. Endothelial dysfunction and decreased production of nitric oxide in the intrahepatic microcirculation of cirrhotic rats. Hepatology 1998;28:926-31.

19 Rockey DC, Chung JJ. Reduced nitric oxide production by endothelial cells in cirrhotic rat liver: endothelial dysfunction in portal hypertension. Gastroenterology 1998;114:344-51.

20 Abraldes JG, Rodríguez-Vilarrupla A, Graupera M, et al. Simvastatin treatment improves liver sinusoidal endothelial dysfunction in $\mathrm{CCl} 4$ cirrhotic rats. J Hepatol 2007;46:1040-6.

21 Trebicka J, Hennenberg M, Laleman W, et al. Atorvastatin lowers portal pressure in cirrhotic rats by inhibition of RhoA/Rho-kinase and activation of endothelial nitric oxide synthase. Hepatology 2007;46:242-53.

22 Chong L-W, Hsu Y-C, Lee T-F, et al. Fluvastatin attenuates hepatic steatosis-induced fibrogenesis in rats through inhibiting paracrine effect of hepatocyte on hepatic stellate cells. BMC Gastroenterol 2015;15:22.

23 Zafra C, Abraldes JG, Turnes J, et al. Simvastatin enhances hepatic nitric oxide production and decreases the hepatic vascular tone in patients with cirrhosis. Gastroenterology 2004;126:749-55.

24 Cash WJ, O'Neill S, O'Donnell ME, et al. Randomized controlled trial assessing the effect of simvastatin in primary biliary cirrhosis. Liver Int 2013;33:1166-74.

25 Pollo-Flores P, Soldan M, Santos UC, et al. Three months of simvastatin therapy vs. placebo for severe portal hypertension in cirrhosis: a randomized controlled trial. Dig Liver Dis 2015;47:957-63.

26 Bang UC, Benfield T, Bendtsen F. Reduced risk of decompensation and death associated with use of statins in patients with alcoholic 
cirrhosis. A nationwide case-cohort study. Aliment Pharmacol Ther 2017;46:673-80.

27 Rodríguez S, Raurell I, Torres-Arauz M, et al. A nitric oxide-donating statin decreases portal pressure with a better toxicity profile than conventional statins in cirrhotic rats. Sci Rep 2017;7:40461.

28 Bishnu S, Ahammed SM, Sarkar A, et al. Effects of atorvastatin on portal hemodynamics and clinical outcomes in patients with cirrhosis with portal hypertension: a proof-of-concept study. Eur $J$ Gastroenterol Hepatol 2018;30:54-9.

29 Simon TG, Bonilla H, Yan P, et al. Atorvastatin and fluvastatin are associated with dose-dependent reductions in cirrhosis and hepatocellular carcinoma, among patients with hepatitis $C$ virus: results from ERCHIVES. Hepatology 2016;64:47-57.

30 Pose E, Trebicka J, Mookerjee RP, et al. Statins: old drugs as new therapy for liver diseases? J Hepatol 2019;70:194-202.

31 Geyer PE, Kulak NA, Pichler G, et al. Plasma proteome profiling to assess human health and disease. Cell Syst 2016;2:185-95.

32 Geyer PE, Wewer Albrechtsen NJ, Tyanova S, et al. Proteomics reveals the effects of sustained weight loss on the human plasma proteome. Mol Syst Biol 2016;12:901.

33 Kulak NA, Geyer PE, Mann M. Loss-less Nano-fractionator for high sensitivity, high coverage proteomics. Mol Cell Proteomics 2017:16:694-705.

34 Kulak NA, Pichler G, Paron I, et al. Minimal, encapsulated proteomicsample processing applied to copy-number estimation in eukaryotic cells. Nat Methods 2014;11:319-24.

35 Cox J, Mann M. MaxQuant enables high peptide identification rates, individualized p.p.b.-range mass accuracies and proteome-wide protein quantification. Nat Biotechnol 2008;26:1367-72.

36 Tyanova S, Temu T, Sinitcyn P, et al. The Perseus computational platform for comprehensive analysis of (prote)omics data. Nat Methods 2016;13:731-40.

$37 \mathrm{Di}$ Costanzo A, Belardinilli F, Bailetti D, et al. Evaluation of polygenic determinants of non-alcoholic fatty liver disease (NAFLD) by a candidate genes resequencing strategy. Sci Rep 2018;8:3702.

38 Dongiovanni P, Valenti L. Genetics of nonalcoholic fatty liver disease. Metabolism 2016;65:1026-37.

39 Wagner A, Regev A, Yosef N. Revealing the vectors of cellular identity with single-cell genomics. Nat Biotechnol 2016;34:1145-60.
40 Snyder MW, Kircher M, Hill AJ, et al. Cell-free DNA comprises an in vivo nucleosome footprint that informs its Tissues-Of-Origin. Cell 2016;164:57-68.

41 European Association for the Study of the Liver. Electronic address: easloffice@easloffice.eu, European Association for the Study of the Liver. EASL clinical practice guidelines for the management of patients with decompensated cirrhosis. J Hepatol 2018;69:406-60.

42 Tandon $\mathrm{P}$, Tangri N, Thomas $\mathrm{L}$, et al. A rapid bedside screen to predict unplanned hospitalization and death in outpatients with cirrhosis: a prospective evaluation of the clinical frailty scale. Am J Gastroenterol 2016;111:1759-67.

43 Kammerlind A-SC, Fristedt S, Ernsth Bravell M, et al. Test-retest reliability of the Swedish version of the Life-Space assessment questionnaire among community-dwelling older adults. Clin Rehabil 2014;28:817-23.

44 Rasmussen ST, Andersen JT, Nielsen TK, et al. Simvastatin and oxidative stress in humans: a randomized, double-blinded, placebocontrolled clinical trial. Redox Biol 2016;9:32-8.

45 Sue MJ, Lee EW, Saab S, et al. Transjugular liver biopsy: safe even in patients with severe coagulopathies and multiple biopsies. Clin Trans/ Gastroenterol 2019;10:e00063.

46 Riaz H, Khan AR, Khan MS, et al. Meta-Analysis of placebocontrolled randomized controlled trials on the prevalence of statin intolerance. Am J Cardiol 2017;120:774-81.

47 Singh S, Singh PP, Singh AG, et al. Statins are associated with a reduced risk of hepatocellular cancer: a systematic review and metaanalysis. Gastroenterology 2013;144:323-32.

48 Zheng Y-X, Zhou P-C, Zhou R-R, et al. The benefit of statins in chronic hepatitis $C$ patients: a systematic review and meta-analysis. Eur J Gastroenterol Hepatol 2017;29:759-66.

49 International Committee of Medical Journal Editors. Uniform requirements for manuscripts submitted to biomedical journals. JAMA 1997;277:927-34.

50 Kaplan DE, Serper MA, Mehta R, et al. Effects of hypercholesterolemia and statin exposure on survival in a large national cohort of patients with cirrhosis. Gastroenterology 2019;156:1693-706. 